\title{
A hybrid finite-difference/lowrank solution to anisotropy acoustic wave equations
}

\author{
Zhen-dong Zhang[1], Tariq Alkhalifah[1] and Zedong Wu[1] \\ 1 Department of Physical Science and Engineering, \\ King Abdullah University of Science and Technology, \\ Thuwal 23955-6900, Saudi Arabia.
}

Email: zhendong.zhang@kaust.edu.sa

(November 26, 2018)

Running head: Quasi-P wavefield extrapolation 


\begin{abstract}
$\mathrm{P}$-wave extrapolation in anisotropic media suffers from SV-wave artifacts and computational dependency on the complexity of anisotropy. The anisotropic pseudo-differential wave equation cannot be solved using an efficient time-domain finite-difference scheme directly. The wavenumber domain allows us to handle pseudo-differential operators accurately, however, requires either smoothly varying media or more computational resources. In the limit of elliptical anisotropy, the pseudo-differential operator reduces to a conventional operator. So, we propose a hybrid-domain solution, which includes a space-domain finite-difference solver for the elliptical anisotropic part of the anisotropic operator and a wavenumber-domain lowrank scheme to solve the pseudo-differential part. Thus, we split the original pseudodifferential operator into a second-order differentiable background and a pseudo-differential correction term. The background equation is solved using the efficient finite-difference scheme and the correction term is approximated by the lowrank approximation. As a result, the correction wavefield is independent of the velocity model, and thus, has reduced rank compared with the full operator. The total computation cost of the proposed method includes the cost of solving a spatial finite-difference time-step update plus several fast Fourier transforms (FFT) related to the rank. The accuracy of the proposed method is of the order of the finite-difference scheme. Applications to a simple homogeneous titled transverse isotropic medium (TTI) and modified BP TTI models demonstrate the effectiveness of the approach.
\end{abstract}

Keywords: Seismic anisotropy, Finite-difference, Lowrank approximation, Wave propagation, Numerical modeling. 


\section{INTRODUCTION}

For realistic seismic wave propagation simulations, we can not ignore the effects of seismic anisotropy. To improve computational efficiency in the case where we are interested in Pwaves only, the acoustic approximation introduced by Alkhalifah (1998) has been an efficient and simple way to have anisotropy effects included in seismic data processing such as Revere Time Migration (RTM) and Full Waveform Inversion (FWI) in the past decades. Pseudoacoustic wave equations based on the acoustic-anisotropy approximation can be solved more efficiently than elastic wave equations. Wave-equation based processing methods including RTM and FWI benefit from this property. Besides, acoustic wavefields are scalars which are easier to process compared to vector wavefields obtained from solving elastic wave equations. However, Finite-difference (FD) solutions to the fourth-order pseudo-acoustic equations suffer from SV-wave artifacts and become unstable for negative $\eta$ (Grechka et al., 2004). Other popular numerical solutions such as the spectral method are free of artifacts and instabilities. However, their computational cost is much larger than FD schemes, which prevents their practical use in industrial applications. Still, finding an efficient and accurate numerical scheme for solving the acoustic anisotropic equation is an ongoing work.

Numerical solutions for pseudo-acoustic wave equations have been thoroughly investigated since the introduction of acoustic anisotropy approximation. Alkhalifah (2000) proposed that by placing the source in isotropic regions, the SV-wave artifacts can be reduced. Since then many other methods including generalized-screen propagators (Han and Wu, 2003), the auxiliary functions (Zhou et al., 2006), the decoupled method (Liu et al., 2009), the finite-difference method (Operto et al., 2009; Liu and Sen, 2010), the energy conservation approach (Zhang et al., 2011), the mixed-domain solution (Zhan et al., 2013), 
the lowrank approximation (Fomel et al., 2013; Song et al., 2013; Wu and Alkhalifah, 2014; Sun et al., 2015) and recently proposed decomposition method (Xu and Zhou, 2014; Zhang and Alkhalifah, 2016; Zhang et al., 2017; Wu and Alkhalifah, 2017) were introduced to solve the pseudo-acoustic wave equations. Among all these methods, the auxiliary function method can be unstable when TTI media are complex (Fletcher et al., 2008); the lowrank approximation can provide stable solutions with high-accuracy but its computational cost is unaffordable for complex media (Fomel et al., 2013). An efficient way to reduce the dependency of the computational cost on model anisotropy complexity is to decompose the total extrapolated wavefield into an isotropic background part and a correction term (Alkhalifah et al., 2013; Xu and Zhou, 2014; Zhang et al., 2017). The background wavefield can be calculated using any conventional numerical method, and then the correction term is calculated using the current wavefield and anisotropic parameters. Using an effective-isotropic model to represent the complex anisotropy for wavefield extrapolation provides a way to reduce the dependency of the computational cost on the level of anisotropy. The decomposition method uses the spatial derivations of the wavefield to approximate the phase angles which is insufficient for multiple wavefronts at a point.

Instead of splitting the wavefield, we propose to decompose the acoustic dispersion relation into a governing relation and a correction term. The governing equation is close to the actual one and plays a dominant role in the solution. It can be numerically solved using the finite-difference method (Zhan et al., 2013; Wu and Alkhalifah, 2018) and the correction term can be solved using the lowrank approximation. The influence of the correction term is significantly smaller than the governing term in the wavefield simulation. Without the correction term, the simulated wavefields are simplified by ignoring some anisotropy effects (Zhang et al., 2005). For each time step, we calculate the background wavefield in the space 
domain and then apply corrections in the wavenumber domain using the lowrank approximation. Because of the lower-accuracy finite-difference solution, we can use fewer ranks to approximate the correction term. Besides, with an appropriately selected background relation, the correction term is independent of the velocity model, which also helps to reduce the rank.

The paper is divided into five parts. After a brief introduction to the developments of popular numerical solutions for pseudo-acoustic wave equations, we give a detailed description of the proposed mix-domain algorithm in the second part. In the third part, we use three different models, which are homogeneous, high-contrast and large-tilt, to verify the effectiveness of the proposed method. We compare the proposed method with the finitedifference and the lowrank approach and also reveal some of its limitations in the fourth part.

\section{THEORY}

Alkhalifah (1998) introduced the acoustic anisotropy approximation by setting the S-wave velocities to zero in the symmetry axis. Simulated wavefields, based on such an approximation, have accurate traveltime and inaccurate amplitudes compared with the original elastic wavefields. For simplicity, we start our analysis by reintroducing the dispersion relation for VTI media, which is given by

$$
k_{z}^{2}=\frac{V_{n}^{2}}{V_{p 0}^{2}}\left(\frac{\omega^{2}}{V_{n}^{2}}-\frac{\omega^{2} k_{h}^{2}}{\omega^{2}-2 V_{n}^{2} \eta k_{h}^{2}}\right),
$$

where $\omega$ is the angular frequency, $k_{z}$ and $k_{h}$ denote the vertical and horizontal wavenumbers, respectively, $V_{p 0}$ and $V_{n}=V_{p 0} \sqrt{1+2 \delta}$ are vertical P-wave and normal moveout (NMO) velocities, and $\eta$ is the ellipticity coefficient. 
The acoustic wave equation corresponding to the dispersion relation has been derived by Alkhalifah (2000), which is written as

$$
\frac{\partial^{2} u}{\partial t^{2}}-\frac{v_{p 0}^{2}}{2}\left((1+2 \epsilon) \frac{\partial^{2}}{\partial h^{2}}+\frac{\partial^{2}}{\partial z^{2}}+Q\right) u=0
$$

where $v_{p 0}$ denotes the vertical P-wave velocity, $\epsilon$ and $\delta$ are Thomsen's parameters. The horizontal Laplacian operator is defined by $\frac{\partial^{2}}{\partial h^{2}}=\frac{\partial^{2}}{\partial x^{2}}+\frac{\partial^{2}}{\partial y^{2}}$, where $\{x, y, z\}$ define the location in Cartesian representation of the space coordinates. Besides, there is a pseudodifferential term in the derived wave equation, which is given by

$$
Q=\sqrt{\left((1+2 \epsilon) \frac{\partial^{2}}{\partial h^{2}}+\frac{\partial^{2}}{\partial z^{2}}\right)^{2}-8(\epsilon-\delta) \frac{\partial^{2}}{\partial h^{2}} \frac{\partial^{2}}{\partial z^{2}}}
$$

The dispersion relation for TTI media with arbitrary tilted angles can be achieved by a rotation of the $z$-axis in the $2 \mathrm{D}$ medium. The wavenumbers with tilted angles, $\theta$, are given by

$$
\begin{aligned}
& \hat{k}_{x}^{2}=\cos ^{2} \theta k_{x}^{2}+\sin 2 \theta k_{x} k_{z}+\sin ^{2} \theta k_{z}^{2}, \\
& \hat{k}_{z}^{2}=\sin ^{2} \theta k_{x}^{2}-\sin 2 \theta k_{x} k_{z}+\cos ^{2} \theta k_{z}^{2} .
\end{aligned}
$$

The corresponding quasi-P wave equation for TTI media in a $2 \mathrm{D}$ medium is given by replacing the original differential operator (equation 2) with the tilted ones (equation 4), which is given by

$$
\frac{\partial^{2} u}{\partial t^{2}}-\frac{v_{0}^{2}}{2}\left(\frac{\partial^{2}}{\partial x^{2}}+\frac{\partial^{2}}{\partial z^{2}}+2 \epsilon\left(\cos ^{2} \theta \frac{\partial^{2}}{\partial x^{2}}+\sin 2 \theta \frac{\partial}{\partial x} \frac{\partial}{\partial z}+\sin ^{2} \theta \frac{\partial^{2}}{\partial z^{2}}\right)+\hat{Q}\right) u=0
$$

with $\hat{Q}=\left\{\frac{\partial^{2}}{\partial x^{2}}+\frac{\partial^{2}}{\partial z^{2}}+2 \epsilon\left(\cos ^{2} \theta \frac{\partial^{2}}{\partial x^{2}}+\sin 2 \theta \frac{\partial}{\partial x} \frac{\partial}{\partial z}+\sin ^{2} \theta \frac{\partial^{2}}{\partial z^{2}}\right)-\right.$

$\left.8(\epsilon-\delta)\left(\cos ^{2} \theta \frac{\partial^{2}}{\partial x^{2}}+\sin 2 \theta \frac{\partial}{\partial x} \frac{\partial}{\partial z}+\sin ^{2} \theta \frac{\partial^{2}}{\partial z^{2}}\right)\left(\sin ^{2} \theta \frac{\partial^{2}}{\partial x^{2}}-\sin 2 \theta \frac{\partial}{\partial x} \frac{\partial}{\partial z}+\cos ^{2} \theta \frac{\partial^{2}}{\partial z^{2}}\right)\right\}^{1 / 2}$

In our proposed method, the dispersion relation in equation 5 is separated into a second- 
order background plus a pseudo-differential correction term, which is given by

$$
\frac{\partial^{2} u}{\partial t^{2}}-v_{0}^{2}(1+C)\left(\frac{\partial^{2}}{\partial x^{2}}+\frac{\partial^{2}}{\partial z^{2}}+2 \epsilon\left(\cos ^{2} \theta \frac{\partial^{2}}{\partial x^{2}}+\sin 2 \theta \frac{\partial}{\partial x} \frac{\partial}{\partial z}+\sin ^{2} \theta \frac{\partial^{2}}{\partial z^{2}}\right)\right) u=0
$$

with $C=\frac{1}{2} \frac{\hat{Q}}{\frac{\partial^{2}}{\partial x^{2}}+\frac{\partial^{2}}{\partial z^{2}}+2 \epsilon\left(\cos ^{2} \theta \frac{\partial^{2}}{\partial x^{2}}+\sin 2 \theta \frac{\partial}{\partial x} \frac{\partial}{\partial z}+\sin ^{2} \theta \frac{\partial^{2}}{\partial z^{2}}\right)}-\frac{1}{2}$, where $C$ is the correction term.

We solve the background part using the finite-difference approximation and the correction term using the lowrank approximation. Note the fact that the correction term is independent of the velocity model which helps to reduce its rank. Besides, the accuracy of the proposed method is determined by the finite-difference scheme and thus the accuracy of lowrank approximation does not need to be high.

The correction term, $C$, cannot be solved using the space-domain finite-difference scheme, but can be solved easily in the wavenumber domain. The lowrank approximation, which has been widely used in solving wave extrapolation problems, provides a feasible way to solve the correction term. Instead of approximating the wave propagation matrix, we utilize it to approximate the correction term. For a certain time step, the correction operator can be approximated by a separable representation,

$$
C(\mathbf{x}, \mathbf{k}) \approx \sum_{m=1}^{M} \sum_{n=1}^{N} C\left(\mathbf{x}, \mathbf{k}_{m}\right) a_{m n} C\left(\mathbf{x}_{n}, \mathbf{k}\right)
$$

where $M$ and $N$ denote the number of selected representative wavenumbers and spatial points in the medium parameters, respectively.

In each time step, after obtaining the spatial finite-difference wavefield for the background medium $(u)$, we transform it into the wavenumber domain and apply the correction,

$$
\triangle u \approx \sum_{m=1}^{M} C\left(\mathbf{x}, \mathbf{k}_{m}\right)\left(\sum_{n=1}^{N} a_{m n}\left(\int \widehat{u}(\mathbf{k}) C\left(\mathbf{x}_{n}, \mathbf{k}\right) d \mathbf{k}\right)\right) .
$$

where $\triangle u$ is the correction wavefield, which can be added to the background wavefield to approximate the exact wavefield in TTI media. The forward extrapolation requires 1 
forward FFT and $N$ inverse FFT in each time step and its adjoint operation (required by RTM) requires $N$ forward FFT and 1 inverse FFT.

In summary, for each time step, the proposed method includes the following steps:

1. Solve the second-order terms of the equation 6 using the conventional spatial finitedifference scheme.

2. Calculate the correction term by the lowrank approximation (equations 7 and 8).

3. Add the correction wavefield to the background wavefield for the next time step.

Diminishing dispersion and preserving stability are critical for wavefield simulations using the FD scheme. We plot the dispersion curves for the background wave equation (equation 6 without the correction term) as shown in Figure 1. In the plots, constant $\eta=0.2$ and $\theta=45^{\circ}$ are used. Wavenumbers are given by $k=\sqrt{k_{z}^{2}+k_{x}^{2}}, k_{z}=k_{x}$. Higherorder finite-difference can suppress the dispersion errors caused by the insufficient number of samples per wavelength. The correction term in equation 6 is solved using the lowrank approximation, which is assumed to be accurate and is free of numerical dispersion. In our proposed method, the numerical implementation of the background wave equation needs to comply with the Courant-Friedrichs-Lewy (CFL) condition (Bakker and Duveneck, 2011). The correction term is independent of time sampling and velocity and we assume that it will not change the stability criterion for the proposed method. Since the wave extrapolation is solved using the FD scheme, we can adapt the boundary conditions used for regular FD schemes to the proposed method. In this paper, we apply the attenuating absorbing boundary condition (Kosloff and Kosloff, 1986) to absorb unwanted reflections from the boundaries. However, the attenuating absorbing boundary condition is imperfect, especially 
for large incident angles (Rao et al., 2016). The more advanced perfectly matched layer absorbing boundary condition (Komatitsch and Tromp, 2003) could be a better choice. However, it is beyond the scope of this paper.

\section{NUMERICAL EXAMPLES}

In the following tests, we extrapolate the background wavefield using 2nd-order in time and 4th-order in space finite-difference schemes. The correction term (equation 8) is approximated by the lowrank approximation with a precision $\sim 10^{-3}$.

\section{Homogeneous TTI Model}

A simple homogeneous TTI model is first used to test the proposed method. The vertical $\mathrm{P}$-wave velocity is $2000 \mathrm{~m} / \mathrm{s}$ and the anisotropy parameters are $\epsilon=0.3, \delta=0.1$ and $\theta=45^{\circ}$. The spatial and time sampling of the wavefield are $6.25 \mathrm{~m}$ and $0.5 \mathrm{~ms}$, respectively. The injected source is a Ricker wavelet with a peak frequency of $5 \mathrm{~Hz}$ and is located at the center of the model. Figures $2 \mathrm{a}, 2 \mathrm{~b}$ and $2 \mathrm{c}$ are snapshots of the wavefields at $0.75 \mathrm{~s}$, which are solved using the lowrank approach, the proposed method (equation 6) and their difference, respectively. Here we take the full lowrank method as a reference solution and compare its solution with our proposed solution. The proposed method can simulate the wavefield including anisotropy effects. However, due to the limited precision of the finite-difference scheme, there are some differences between these two solutions. To compare the waveforms, we extract two slices: the horizontal and vertical ones from Figure 3. The waveforms in both solutions are close to each other except their maximum amplitudes. 


\section{High-contrast TTI Model}

We use part of the BP TTI model to verify the effectiveness of our proposed method for high-contrast models. The model as shown in Figure 4 has low velocities in the shallow sedimentary region and high velocities for the salt body. A Ricker wavelet with a peak frequency of $15 \mathrm{~Hz}$ is placed at center-top of the model. The spatial and time sampling used for simulation are $6.25 \mathrm{~m}$ and $0.5 \mathrm{~ms}$, respectively. These parameters are delicately selected to satisfy the CFL condition and minimize the dispersion error. Two snapshots at $1 \mathrm{~s}$ and $2 \mathrm{~s}$ are shown in Figures $5 \mathrm{a}$ and $5 \mathrm{~b}$, respectively. Simulated wavefields travel through the salt bodies without apparent numerical errors. A high-order FD scheme (2ndand 16th-order in time and space, respectively) and a more precise lowrank approximation $\left(\sim 10^{-5}\right)$ is used as the reference solution. We set the source in the center of the model to avoid potential influences from the imperfect absorbing boundaries. we first compare two complex wavefields of reference solution and proposed schemes as Figures $6 \mathrm{a}$ and $6 \mathrm{~b}$ indicate. Their difference shown in Figure 6c is ignorable. We then extract two profiles (dashed lines in Figures 6a) from the snapshots as shown in Figure 7. Their differences in horizontal (Figures $7 \mathrm{a}$ and $7 \mathrm{c}$ ) and vertical directions (Figures $7 \mathrm{~b}$ and $7 \mathrm{~d}$ ) are $\sim 10^{-3}$ order smaller than the reference solution. Long-time recorded (7.5 s) common-shot-gathers (CSG) in Figure 8 verify the stability of the proposed method for a large recording time. Representative arrivals including reflections from the sea bottom and salt top can be detected. However, there are unwanted reflections from the imperfect boundaries as discussed in the Theory section. Their difference (Figure $8 \mathrm{c})$ is still ignorable $\left(\sim 10^{-2}\right.$ smaller than the reference solution). 


\section{Modified BP TTI Model}

We further verify the effectiveness of our proposed method using the modified BP TTI model shown in Figure 9. The vertical velocity model (Figure 9a) is smoother and also has fewer interfaces than anisotropy parameters (Figures 9c and 9d). A Ricker wavelet with a peak frequency of $25 \mathrm{~Hz}$ is injected as a point source. Space and time sampling of the wavefield are $6.25 \mathrm{~m}$ and $0.5 \mathrm{~ms}$, respectively. In this example, we first compare two complex wavefields calculated by the anisotropic lowrank method and our proposed method. Solutions of the original lowrank method are considered as reference solutions in this example (Fomel et al., 2013). As Figure 10 shows, the source is located at the center of the model and the two wavefields are similar to each other. We also take two profiles (dashed lines in Figure 10a) of the wavefields for a clear comparison as shown in Figure 11. It shows that the simulated wavefield using our proposed method are close to the one from lowrank approximations. The proposed numerical scheme can be used as the modeling engine for Reverse Time Migration (Etgen et al., 2009).

\section{DISCUSSIONS}

The proposed method is a combination of the high-efficiency finite-difference method and the high-accuracy spectral methods. It can simulate anisotropic wavefields without the artifacts often faced in the finite-difference approach and also can reduce the unaffordable computational cost faced by spectral approaches. A fair comparison between the finitedifference approach, the lowrank approximation and the proposed method is provided in Table 1. For each time step, with a model size of $N_{x}$, the number of floating-point operations for the lowrank approach is $\mathcal{O}\left(M N_{x} \log N_{x}\right)$, where $M$ is the number of fast Fourier 
transforms (FFT) and a typical value for BP TTI model is 10 (Fomel et al., 2013). For our proposed method, the number of floating-point operations is $\mathcal{O}\left(L N_{x}+K N_{x} \log N_{x}\right)$. $L$ is the size of the FD stencil and a typical $K$ equals 3 for acceptable accuracy. The accuracy of the simulated wavefields is decided by the number of points $(L)$ used for finitedifference and the ranks $(K)$ used in lowrank approximation. The proposed method applies to general pseudo-equations with mild modifications to the governing equation and its corresponding corrections. Besides, the proposed method can utilize the same well established numerical techniques (such as the absorbing boundaries) applied to regular finite-difference approaches. The pseudo-acoustic wave equation based on an acoustic-Earth assumption has limitations in practice. However, it is a reasonable approximation for marine and land data without strong converted waves. The real Earth has too many physical processes to be

considered in numerical simulations. As a result, most processing methods such as FWI are utilizing simplified physics in wavefield extrapolation. Inline with the simplification, traveltime or phases instead of amplitudes are used in matching the observed and simulated data, which means the proposed numerical solution can be used as a modeling engine in FWI (Wang and Tsvankin, 2016; Li et al., 2017; Zhang and Alkhalifah, 2017).

\section{CONCLUSIONS}

We present a mixed-domain solution to the pseudo-differential acoustic wave equation. The proposed method separates the original dispersion relation into a simple governing relation and its correction corresponding to anisotropy. The governing equation is solved here using the finite-difference approximation and the pseudo-differential corrections are calculated using the lowrank approximation. In each time step, the full anisotropic wavefield is calculated by adding the corrections to the background wavefield. The real feature of 
this method is the application of the finite-difference method, which is free of shear-wave artifacts and can adopt existing numerical schemes used in the finite-difference method. The precision of the simulation is the same as finite-difference schemes, and thus, the required accuracy of the lowrank approximation is kept in the same order with the finitedifference scheme used in the simulation. Besides, the correction term is independent of the velocity model, and thus the total rank is reduced. Numerical examples verify the effectiveness of the proposed method. The proposed method has the same limitation with the acoustic anisotropic approximation in which we can only generate P-wave fields with accurate traveltimes. It can be used as a modeling engine in a phase- or traveltime-objected inverse problem.

\section{ACKNOWLEDGMENTS}

We thank John Etgen, Dimitri Komatitsch, Valentina Socco and three anonymous reviewers for improving the quality of the paper. We thank Hui Wang, Nabil Masmoudi and Yike Liu for their helpful discussions. We thank KAUST for its support and specifically the seismic wave analysis group members for their valuable insights. For computer time, this research used the resources of the Supercomputing Laboratory at King Abdullah University of Science \& Technology (KAUST) in Thuwal, Saudi Arabia. 


\section{REFERENCES}

Alkhalifah, T., 1998, Acoustic approximations for processing in transversely isotropic media: Geophysics, 63, 623-631.

- , 2000, An acoustic wave equation for anisotropic media: Geophysics, 65, 1239-1250.

Alkhalifah, T., X. Ma, U. bin Waheed, and M. Zuberi, 2013, Efficient anisotropic wavefield extrapolation using effective isotropic models: Presented at the 75th EAGE Conference \& Exhibition incorporating SPE EUROPEC 2013.

Bakker, P. M., and E. Duveneck, 2011, Stability analysis for acoustic wave propagation in tilted TI media by finite differences: Geophysical Journal International, 185, 911-921.

Etgen, J., S. H. Gray, and Y. Zhang, 2009, An overview of depth imaging in exploration geophysics: Geophysics, 74, WCA5-WCA17.

Fletcher, R., X. Du, and P. J. Fowler, 2008, A new pseudo-acoustic wave equation for TI media, in SEG Technical Program Expanded Abstracts 2008: Society of Exploration Geophysicists, 2082-2086.

Fomel, S., L. Ying, and X. Song, 2013, Seismic wave extrapolation using lowrank symbol approximation: Geophysical Prospecting, 61, 526-536.

Grechka, V., L. Zhang, and J. W. Rector III, 2004, Shear waves in acoustic anisotropic media: Geophysics, 69, 576-582.

Han, Q., and R.-S. Wu, 2003, One-way dual-domain propagators for scalar P-wave in VTI media, in SEG Technical Program Expanded Abstracts 2003: Society of Exploration Geophysicists, 157-160.

Komatitsch, D., and J. Tromp, 2003, A perfectly matched layer absorbing boundary condition for the second-order seismic wave equation: Geophysical Journal International, 154, 146-153. 
Kosloff, R., and D. Kosloff, 1986, Absorbing boundaries for wave propagation problems: Journal of Computational Physics, 63, 363-376.

Li, V., H. Wang, I. Tsvankin, E. Díaz, and T. Alkhalifah, 2017, Inversion gradients for acoustic VTI wavefield tomography: Geophysics, 82, WA55-WA65.

Liu, F., S. A. Morton, S. Jiang, L. Ni, and J. P. Leveille, 2009, Decoupled wave equations for $\mathrm{P}$ and SV waves in an acoustic VTI media, in SEG Technical Program Expanded Abstracts 2009: Society of Exploration Geophysicists, 2844-2848.

Liu, Y., and M. K. Sen, 2010, Acoustic VTI modeling with a time-space domain dispersionrelation-based finite-difference scheme: Geophysics, 75, A11-A17.

Operto, S., J. Virieux, A. Ribodetti, and J. E. Anderson, 2009, Finite-difference frequencydomain modeling of viscoacoustic wave propagation in $2 \mathrm{D}$ tilted transversely isotropic (TTI) media: Geophysics, 74, T75-T95.

Rao, Y., Y. Wang, Z. Zhang, Y. Ning, X. Chen, and J. Li, 2016, Reflection seismic waveform tomography of physical modelling data: Journal of Geophysics and Engineering, 13, 146.

Song, X., S. Fomel, and L. Ying, 2013, Lowrank finite-differences and lowrank fourier finitedifferences for seismic wave extrapolation in the acoustic approximation: Geophysical Journal International, ggt017.

Sun, J., S. Fomel, and L. Ying, 2015, Low-rank one-step wave extrapolation for reverse time migration: Geophysics, 81, S39-S54.

Wang, H., and I. Tsvankin, 2016, Feasibility of waveform inversion in acoustic orthorhombic media, in SEG Technical Program Expanded Abstracts 2016: Society of Exploration Geophysicists, 311-316.

Wu, Z., and T. Alkhalifah, 2014, The optimized expansion based low-rank method for wavefield extrapolation: Geophysics, 79, T51-T60. 
— 2017 , An efficient Helmholtz solver for acoustic transversely isotropic media: Geophysics, 83, C75-C83.

— , 2018, A highly accurate finite-difference method with minimum dispersion error for solving the Helmholtz equation: Journal of Computational Physics, 365, 350-361.

$\mathrm{Xu}$, S., and H. Zhou, 2014, Accurate simulations of pure quasi-P-waves in complex anisotropic media: Geophysics, 79, T341-T348.

Zhan, G., R. C. Pestana, and P. L. Stoffa, 2013, An efficient hybrid pseudospectral/finitedifference scheme for solving the TTI pure P-wave equation: Journal of Geophysics and Engineering, 10, 025004 .

Zhang, L., J. W. Rector, and G. M. Hoversten, 2005, Finite-difference modelling of wave propagation in acoustic tilted TI media: Geophysical Prospecting, 53, 843-852.

Zhang, Y., H. Zhang, and G. Zhang, 2011, A stable TTI reverse time migration and its implementation: Geophysics, 76, WA3-WA11.

Zhang, Z., and T. Alkhalifah, 2016, Efficient quasi-P wavefield extrapolation using an isotropic lowrank approximation: Presented at the 78th EAGE Conference and Exhibition 2016.

Zhang, Z.-D., and T. Alkhalifah, 2017, Full waveform inversion using oriented time-domain imaging method for vertical transverse isotropic media: Geophysical Prospecting, 65, $166-180$.

Zhang, Z.-d., Y. Liu, T. Alkhalifah, and Z. Wu, 2017, Efficient anisotropic quasi-P wavefield extrapolation using an isotropic low-rank approximation: Geophysical Journal International, 213, 48-57.

Zhou, H., G. Zhang, and R. Bloor, 2006, An anisotropic acoustic wave equation for modeling and migration in 2D TTI media, in SEG Technical Program Expanded Abstracts 2006: 
Society of Exploration Geophysicists, 194-198. 


\section{LIST OF TABLES}

1 A general comparison of numerical solutions. 
Table 1: A general comparison of numerical solutions.

Floating-point numbers Requirements SV-wave Coding complexity

\begin{tabular}{lcccc}
\hline Finite-difference & $\mathcal{O}\left(L N_{x}\right)$ & $\eta>0$ & Yes & Low \\
Lowrank & $\mathcal{O}\left(M N_{x} \log N_{x}\right)$ & None & No & Moderate \\
Proposed & $\mathcal{O}\left(L N_{x}+K N_{x} \log N_{x}\right)$ & None & No & High
\end{tabular}




\section{LIST OF FIGURES}

1 Dispersion errors versus wavenumbers for different FD schemes. For a clear comparison, wavenumbers are defined as $k=\sqrt{k_{z}^{2}+k_{x}^{2}}, k_{z}=k_{x}$.

2 Snapshots of the wavefield at 0.75 s. (a) The lowrank solution, (b) our proposed method and (c) their difference. They're plotted in the same scale and their differences are small.

3 Waveform slices in Figure 2. (a) In horizontal direction and (b) in depth direction. The proposed method provides close simulations to the lowrank solution.

4 Revised BP TTI model with salt bodies. (a) Vertical velocity, (b) tilted angle, (c) $\delta$ and $(\mathrm{d}) \epsilon$.

5 Snapshots at $1 \mathrm{~s}(\mathrm{a})$ and $2 \mathrm{~s}(\mathrm{~b})$. The attenuating absorbing boundary condition is applied at the top of the model.

6 Snapshot comparison. a) Reference solution, b) proposed scheme and c) their difference.

7 Waveform slices from Figure 6. a) Horizontal direction, b) vertical direction, c) and d) are their difference, respectively. The differences in both directions are $\sim 10^{-3}$ smaller than the reference solution.

8 Long time recordings. a) Reference solution, b) proposed scheme and c) their difference. The plotting uses a clip of $99 \%$ for a clear view. Some primary arrivals are recorded as indicated by the arrows. Unwanted reflections from the absorbing boundaries are ignorable. Their difference is $\sim 10^{-2}$ smaller than the reference solution.

9 Revised BP TTI model. (a) Vertical velocity, (b) tilted angle, (c) $\delta$ and (d) $\epsilon$. 
10 Snapshots of the wavefield at $0.75 \mathrm{~s}$. (a) The lowrank solution, (b) our proposed method and (c) their difference. They're plotted in the same scale and their differences are small.

11 Waveform slices in Figure 10. (a) In horizontal direction and (b) in depth direction. The proposed method provides close simulations to the lowrank solution except for the inaccurate magnitudes. 


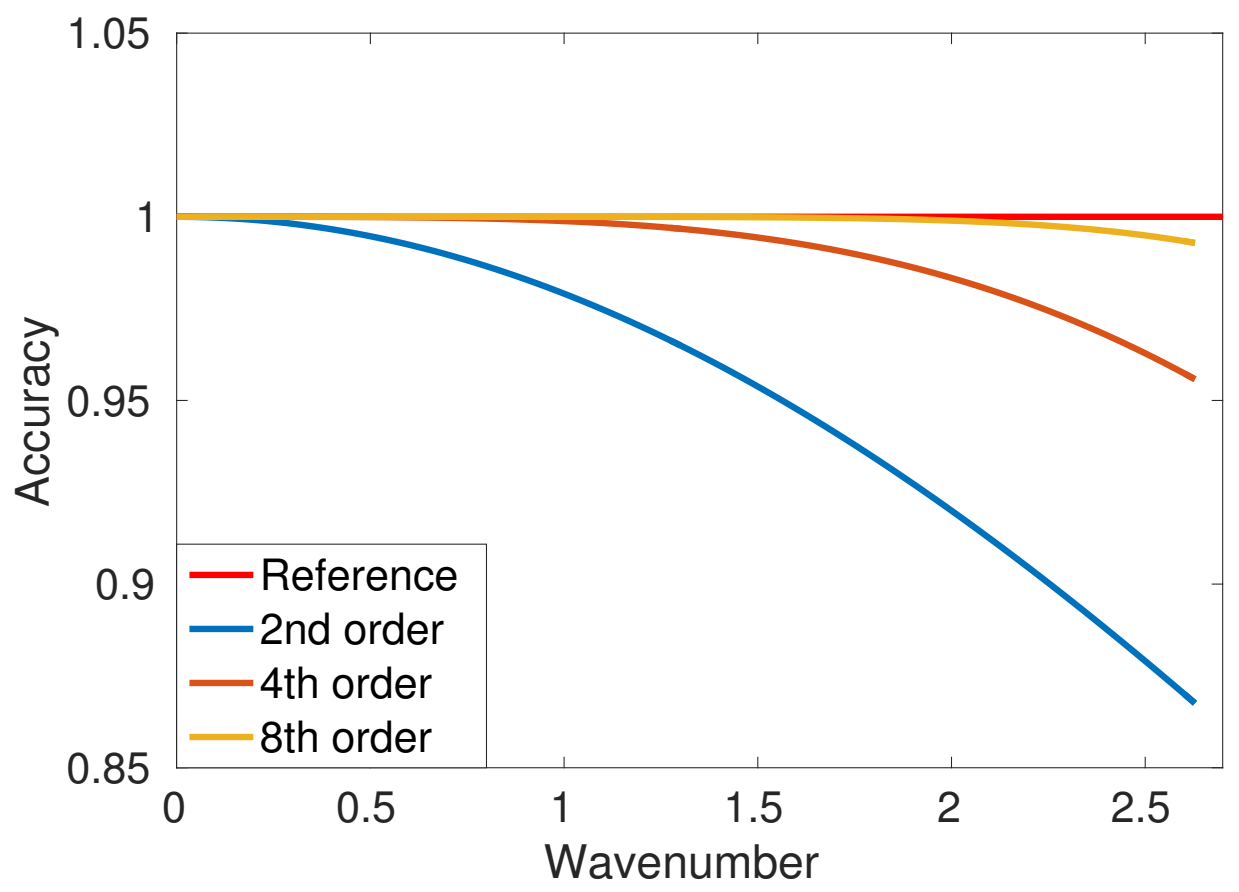

Figure 1: Dispersion errors versus wavenumbers for different FD schemes. For a clear comparison, wavenumbers are defined as $k=\sqrt{k_{z}^{2}+k_{x}^{2}}, k_{z}=k_{x}$. 

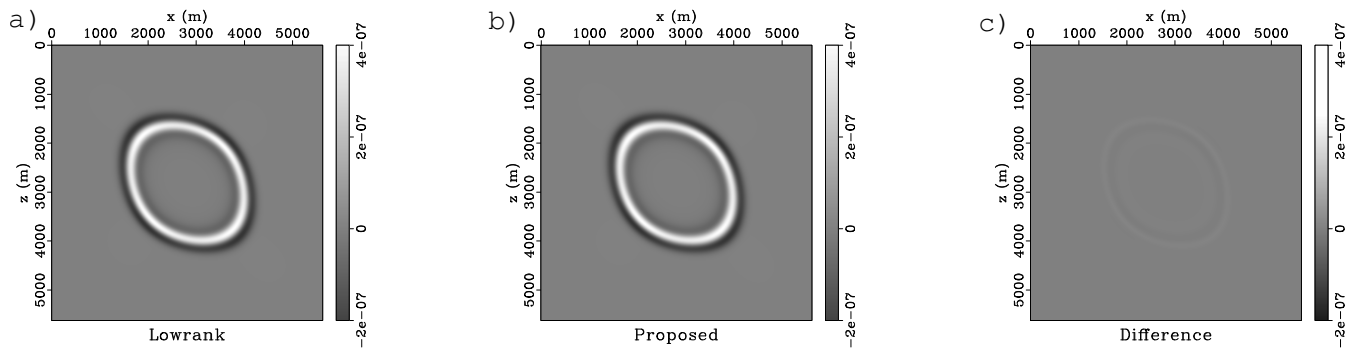

Figure 2: Snapshots of the wavefield at 0.75 s. (a) The lowrank solution, (b) our proposed method and (c) their difference. They're plotted in the same scale and their differences are small. 

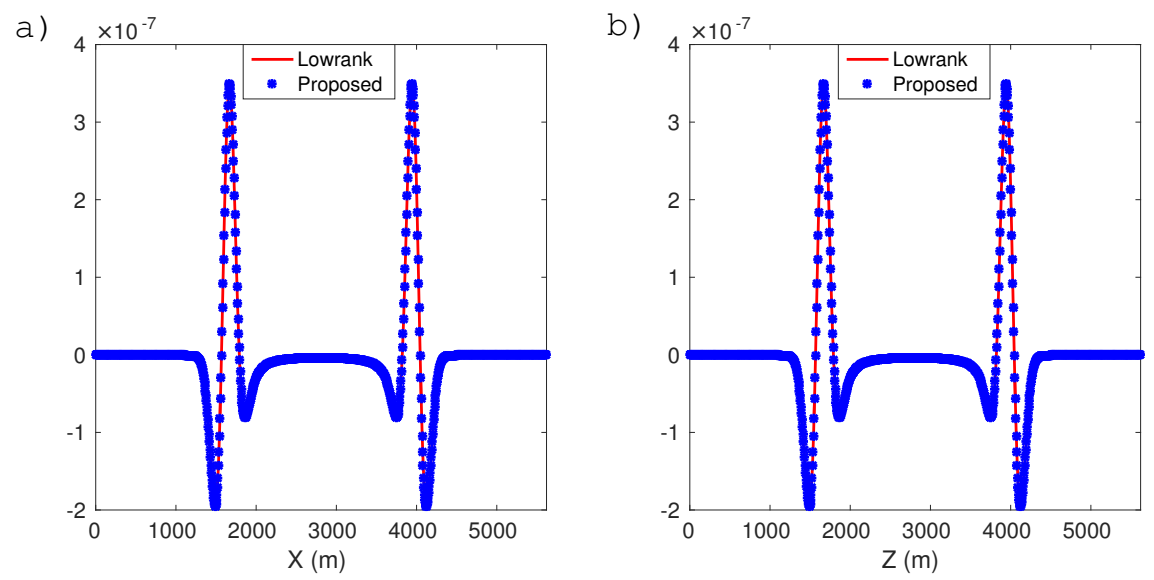

Figure 3: Waveform slices in Figure 2. (a) In horizontal direction and (b) in depth direction. The proposed method provides close simulations to the lowrank solution. 

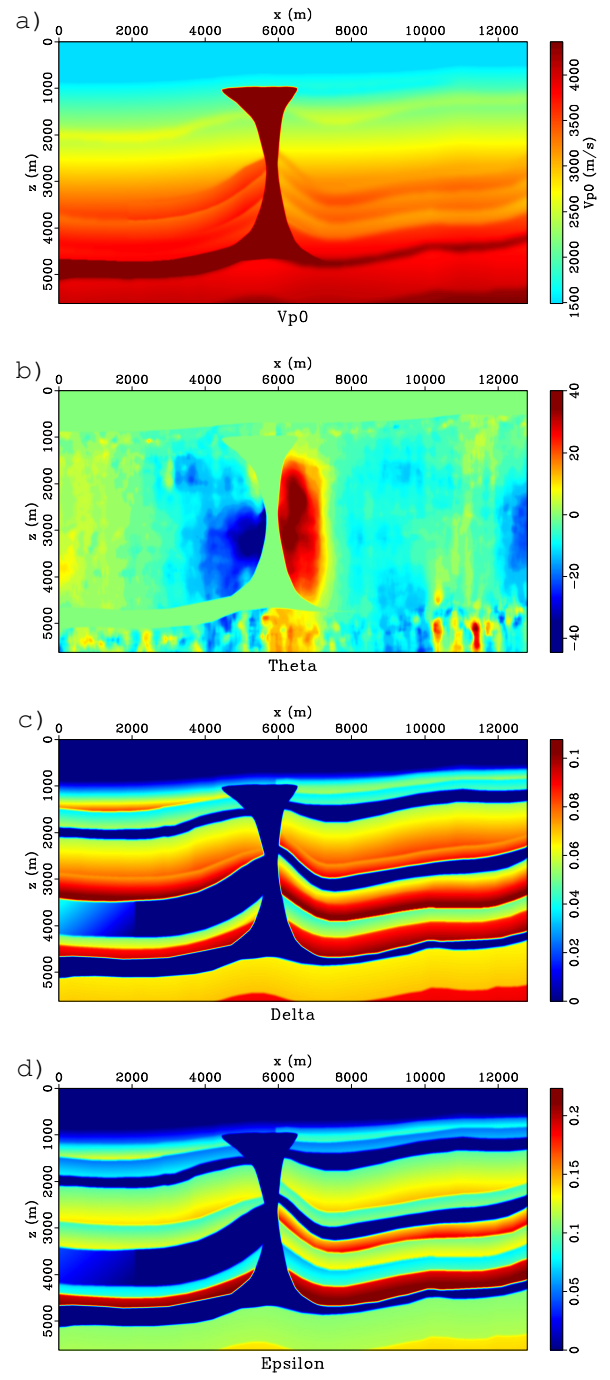

Figure 4: Revised BP TTI model with salt bodies. (a) Vertical velocity, (b) tilted angle, (c) $\delta$ and (d) $\epsilon$. 

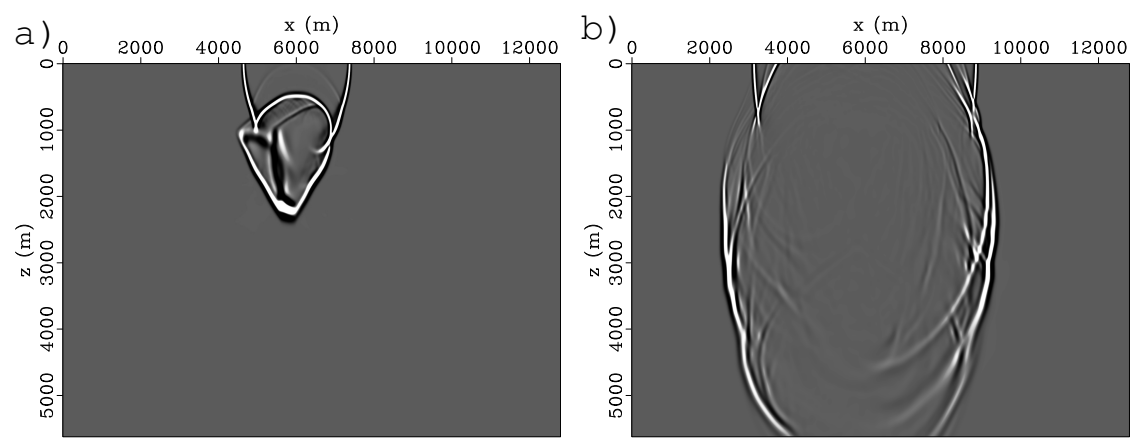

Figure 5: Snapshots at 1s (a) and 2s (b). The attenuating absorbing boundary condition is applied at the top of the model. 

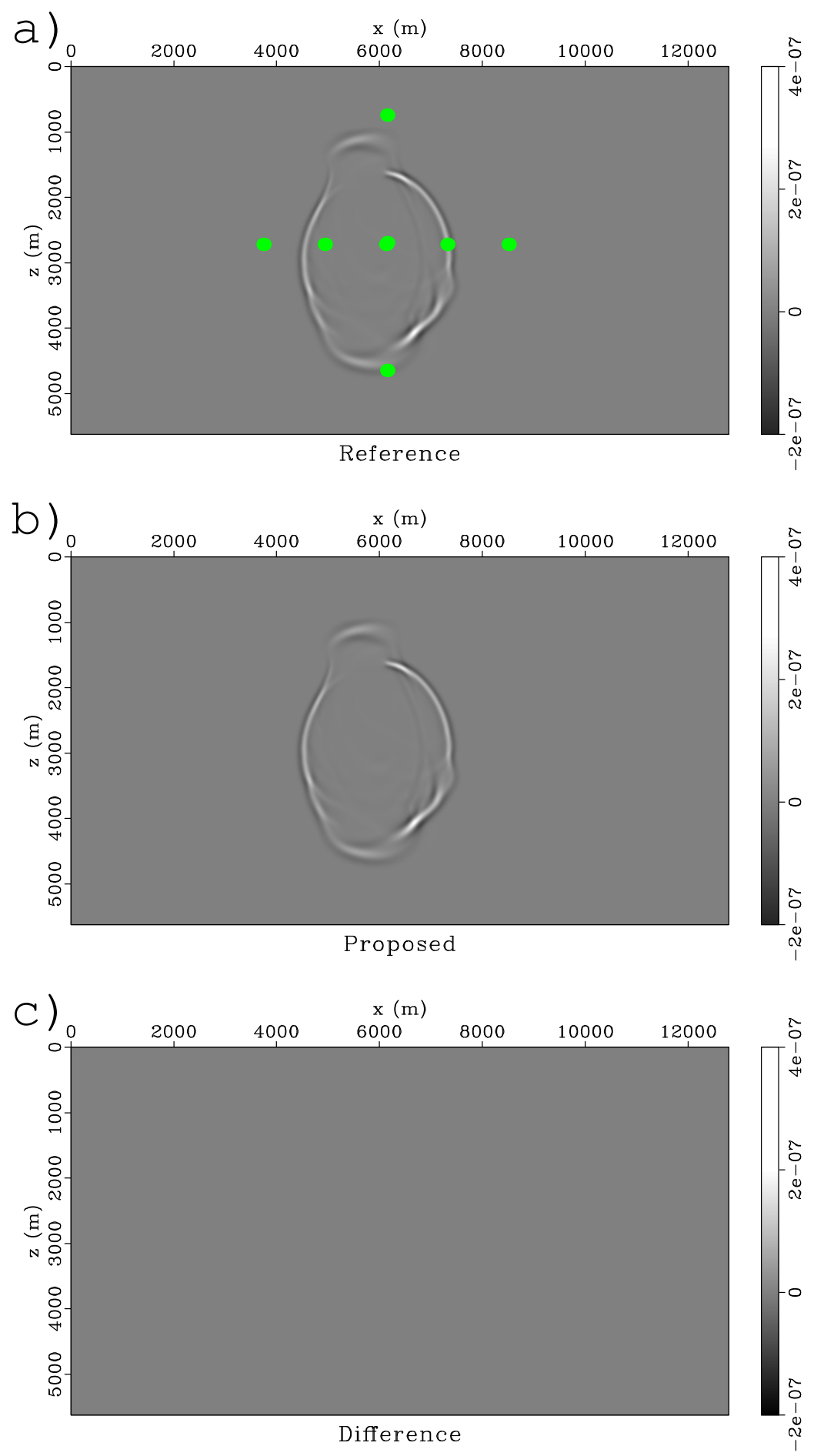

Figure 6: Snapshot comparison. a) Reference solution, b) proposed scheme and c) their difference. 

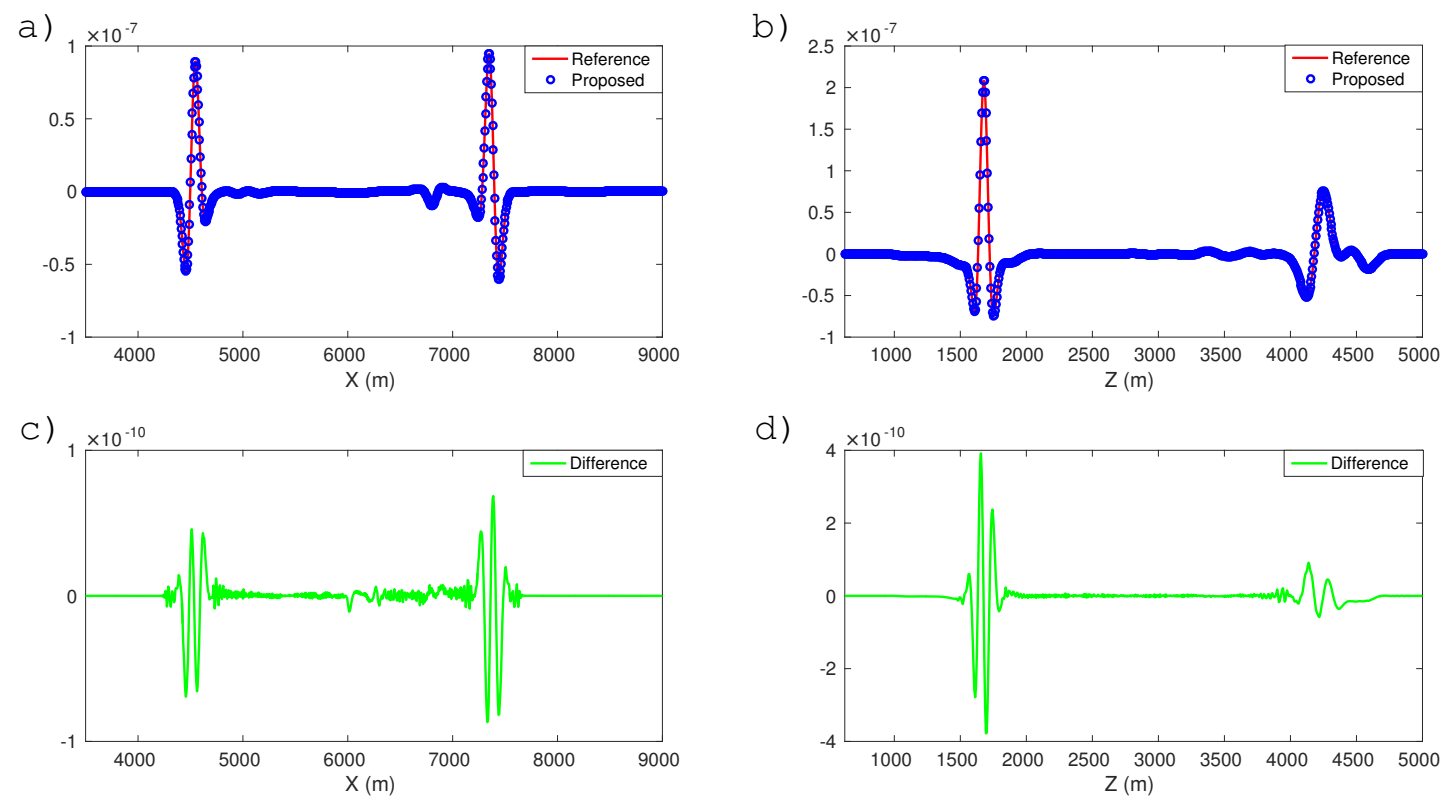

d)

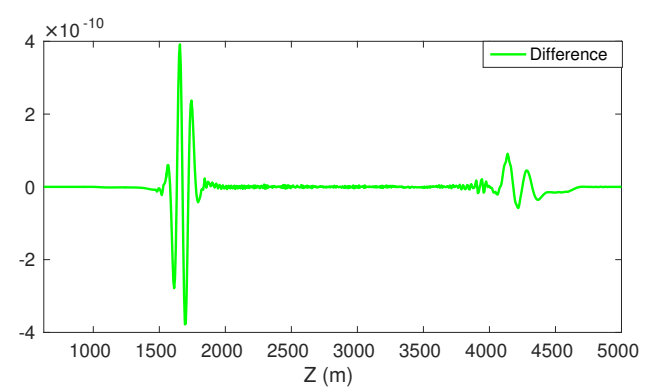

Figure 7: Waveform slices from Figure 6. a) Horizontal direction, b) vertical direction, c) and d) are their difference, respectively. The differences in both directions are $\sim 10^{-3}$ smaller than the reference solution. 

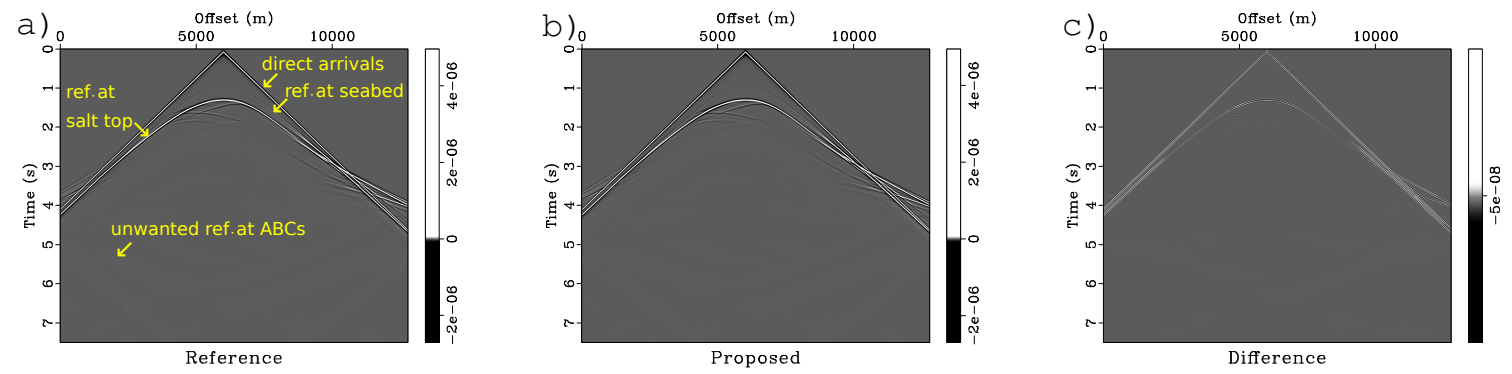

Figure 8: Long time recordings. a) Reference solution, b) proposed scheme and c) their difference. The plotting uses a clip of $99 \%$ for a clear view. Some primary arrivals are recorded as indicated by the arrows. Unwanted reflections from the absorbing boundaries are ignorable. Their difference is $\sim 10^{-2}$ smaller than the reference solution. 

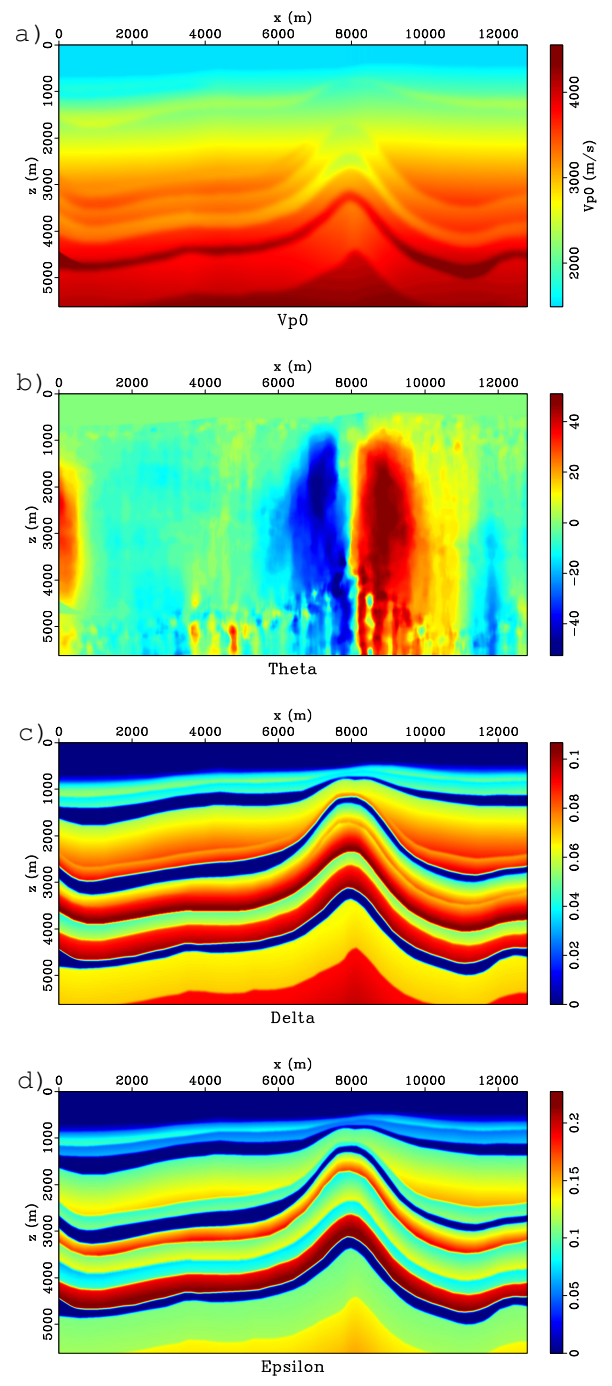

Figure 9: Revised BP TTI model. (a) Vertical velocity, (b) tilted angle, (c) $\delta$ and (d) $\epsilon$. 

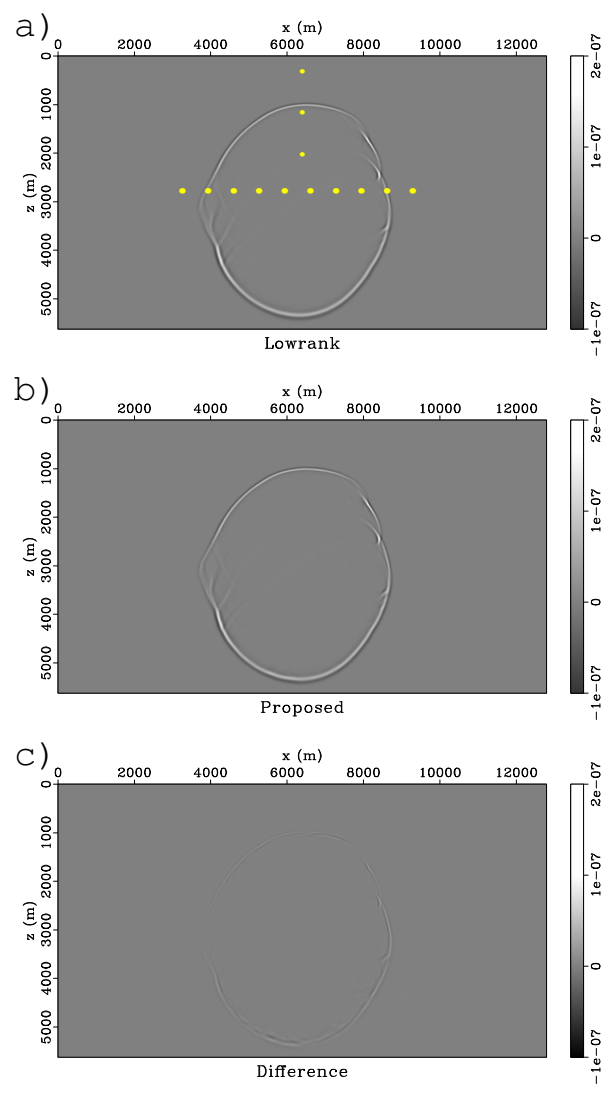

Figure 10: Snapshots of the wavefield at 0.75 s. (a) The lowrank solution, (b) our proposed method and (c) their difference. They're plotted in the same scale and their differences are small. 

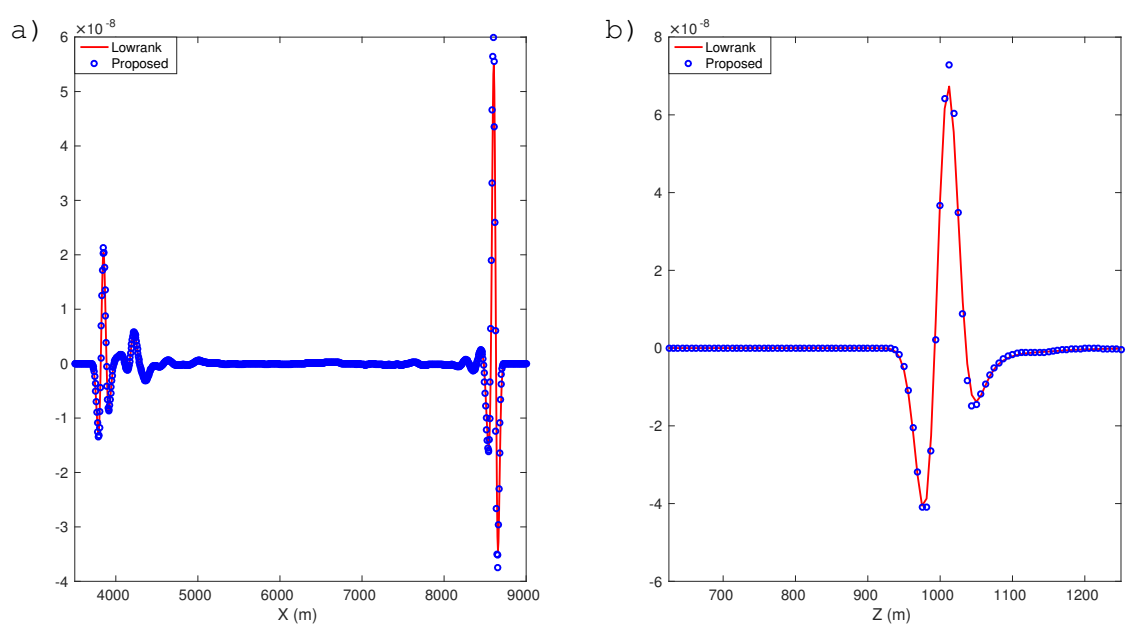

Figure 11: Waveform slices in Figure 10. (a) In horizontal direction and (b) in depth direction. The proposed method provides close simulations to the lowrank solution except for the inaccurate magnitudes. 